Fadhil Y Jassim

BDS, CES, DSO (Asst. Prof.)

Khawla M Awni

BDS, MSc (Lec.)

\section{Dentoskeletal Parameters Comparison of Different Types of Overbite in Class I Occlusion (Lateral Cephalomtric Study)}

\author{
Dept of Pedod, Orthod and Prev Dentistry \\ College of Dentistry, University of Mosul \\ Dept of Pedod, Orthod and Prev Dentistry \\ College of Dentistry, University of Mosul
}

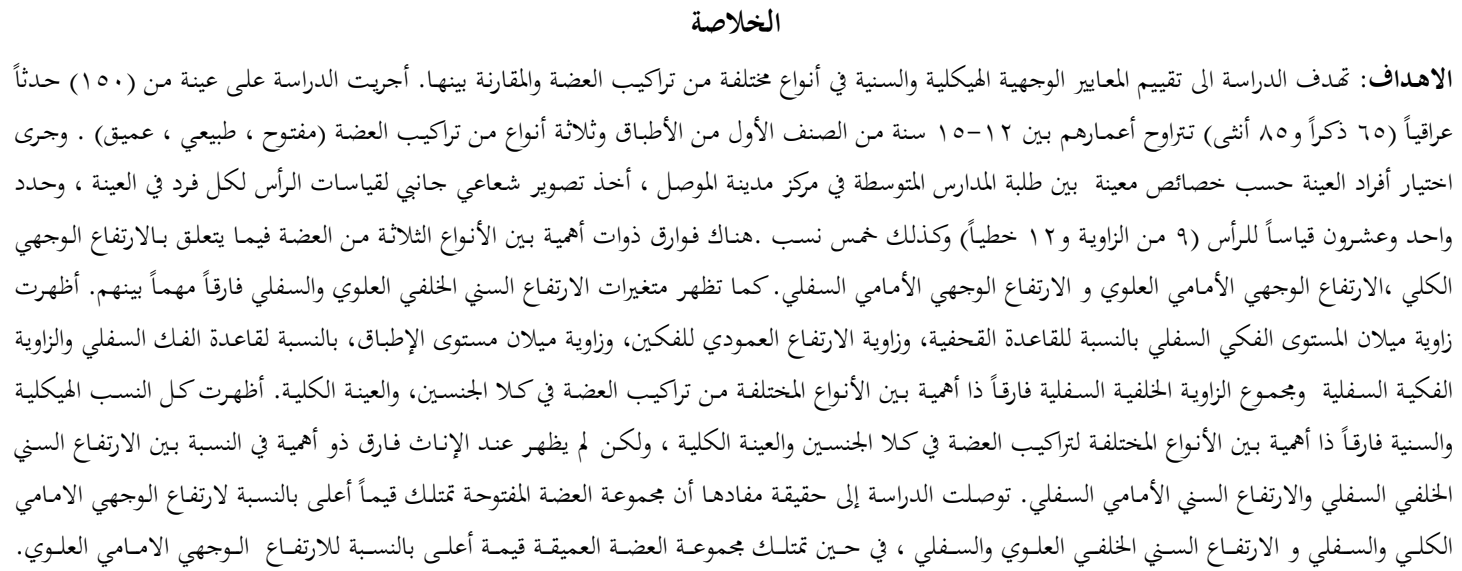

\title{
ABSTRACT
}

Aims: This study is intended to evaluate the skeletofacial and dental parameters in different types of overbite and to compare among them. Materials and Methods: It was carried out on a sample of 150 Iraqi adolescents (65 males and 85 females), aged (12-15) years with class I malocclusion have different overbite (open, normal and deep bites).The sample were selected according to certain criteria among the students of intermediate schools in the center of Mosul city. Lateral cephalometric radiograph was taken for each subject. Twenty-one cephalometric measurements (nine angular and twelve linear) and five ratios had been determined. Results: There are significant differences among the three types of overbite concerning total anterior facial height, upper anterior facial height and lower anterior facial height variables. Also, upper posterior dental height and lower posterior dental height show a significant difference between them. Anterior cranial base to mandibular plane angle, palato mandibular plane angle, occluso-mandibular plane angle, gonial angle and summation of posterior angles are significantly different among the different types of overbite in both sexes and the total sample. All skeletal and dental ratios are significantly different among the three types of overbite in both sexes and total sample. Conclusions: The study concludes that open bite group possesses significant higher values of total and lower anterior facial height, upper and lower posterior dental height, while deep bite group possesses significant higher value of upper anterior facial height.

Key Words: Skeletofacial , Cephalometric.

Jassim FY, Awni KM. Dentoskeletal Parameters Comparison of Different Types of Overbite in Class I Occlusion (Lateral Cephalomtric Study). Al-Rafidain Dent J. 2014; 14(1):52 -59.

Received: 13/9/2012 Sent to Referees: 17/9/2012 Accepted for Publication: 21/11/2012

\section{INTRODUCTION}

Vertical dimension is the most important dimension to the clinical orthodontist and that vertical dysplasias are inseparately related to both open and closed bites. ${ }^{(1)}$ Normal occlusion can be defined as an occlusion which satisfies the requirements of function and aesthetics but in which there are minor irregularities of individual teeth. ${ }^{(1)}$ 
Anterior open bite has been defined as that condition where upper incisor crowns fail to overlap the incisal third of the lower incisor crowns when the mandible is brought into full occlusion, while deep bite is defined as an excessive vertical overlap of the incisor teeth when the molars and premolars are in maximum intercuspation. $^{(2)}$

The relationship of the mandible to the maxilla has long been noted as bearing upon the incisor occlusion, in orthodontic, thorough knowledge of the skeleton and dental components that contribute to particular malocclusion is essential. ${ }^{(3)}$ This study is intended to evaluate cephalometrically the skeletal and dental vertical dimensions in class I Mosul adolescents with different types of overbite,to define the possible differences between the two sexes within each type of overbite,to determine the skeletal and dental variations that could exist between different types of overbite in males, females and the total sample.

\section{MATERIALS AND METHODS}

The sample of this study were carried out on seventeen intermediate schools have been randomly selected within the city of Mosul.The sample consisted of 150 subjects (males and females) selected from the clinical examination of 9945 students, aged 12-15 years old, class I occlusion with different types of overbite.

\section{Criteria of the Samples:}

The subjects were divided into three groups on the basis of over bite. ${ }^{(4)}$ (the over bite was measured on lateral cephalometric radiograph).

1. Open Bite Group:(24 males and 26 females) The overbite being smaller than or equal to $-1 \mathrm{~mm} .^{(5)} 2$. Normal Overbite Group:(25 males and 25 females) The overbite being more than $+2 \mathrm{~mm}$ but less than or equal to $+4 \mathrm{~mm}$.3. Deep Bite Group: (16 males and 34 females)The overbite being more than $+4 \mathrm{~mm}$. All radiographs are taken using S.Swhite cephalometer with a Wehmer cephalostate (Model W-105A), germany.

Lateral cephalometric radiographs were taken for each subject at centric occlusion and the lips were in relaxed position. The head was fixed by two ear rods laterally and a plastic nasal stopper on the bridge of the nose anteriorly. The subjects were in standing position with the Frankfort horizontal parallel to the floor. ${ }^{(6)}$

The cephalometric landmarks and planes are recorded manually from the tracing of the radiographs to obtain the following measurements:

\section{- Skeletal Linear Measurements:}

TAFH-Total Anterior Facial Height (N$\mathrm{Me}$ ): The vertical distance from nasion to menton. ${ }^{(5)}$ UAFH-Upper Anterior Facial Height (N-ANS): The vertical distance from nasion to anterior nasal spine. ${ }^{(7,8)}$ LAFH-Lower Anterior Facial Height (ANS-Me): The vertical distance from anterior nasal spine to menton. ${ }^{(7,8)} \mathrm{TPFH}-$ Total Posterior Facial Height (S-Go): It is the vertical distance from the center of sella turcica to gonion. ${ }^{(7,8)} \mathrm{UPFH}-$ Upper Posterior Facial Height: It is the perpendicular line from point $S$ to palatal plane $.^{(9)} \mathrm{LPFH}-$ Lower Posterior Facial Height: It is the perpendicular line from the point gonion to the palatal plane. ${ }^{(9)}$ RH- Ramus Height (Ar-Go): The distance from point Ar to point Go. ${ }^{(9)}$

\section{$\checkmark$ Dental Linear Measurements:}

UADH-(Upper Anterior Dental Height): The perpendicular distance from upper incisor edge projected at a right angle to the palatal plane. ${ }^{(7,8)}$ UPDH-(Upper Posterior Dental Height):The perpendicular distance from the mesiobuccal cusp of the upper six to the palatal plane. ${ }^{(10)}$ LADH-(Lower Anterior Dental Height):The perpendicular distance from lower central incisor edge projected at a right angle to the MP. ${ }^{(10)} \mathrm{LPDH}-$ (Lower Posterior Dental Height): The perpendicular distance from the mesiobuccal cusp of the lower first molar to the MP. ${ }^{(10)}$

\section{- Skeletal and Dental Ratios:}

TPFH/TAFH: Is the ratio between the total posterior facial height and total anterior facial height. ${ }^{(11)} \mathrm{LAFH} / \mathrm{TAFH}$ : Is the ratio between the lower anterior facial height and total anterior facial height. ${ }^{(12)}$ UAFH/TAFH: Is the ratio between the upper anterior facial height and total anterior facial height. ${ }^{(11)} \mathrm{UPDH} / \mathrm{UADH}$ : Is the ratio between the upper posterior dental height and upper anterior dental 
height. ${ }^{(10)}$ LPDH/LADH: Is the $r$ atio between the lower posterior dental height and lower anterior dental height. ${ }^{(10)}$

\section{$\checkmark$ Angular Measurements:}

SN-MP angle: It represents the inclination of the mandible to the anterior cranial base. ${ }^{(12)}$ PP-MP (Palato Mandibular Plane Angle): The angle of inclination of the mandibular to the maxillary base. ${ }^{(9)} \mathrm{OP}-\mathrm{MP}$ angle (Mandibular Occlusal Angle): This angle is formed between occlusal and mandibular planes. ${ }^{(13)}$ SN-PP angle: The angle of palatal plane inclination in relation to anterior cranial base. ${ }^{(11)}$ OP-PP angle: The angle which is formed by the intersection between occlusal plane and palatal plane. ${ }^{(9)}$ Ar.Go.Me (Gonial Angle): The angle between the posterior border of the ramus (Ar-Go) and lower border of the mandible (Go-Me). ${ }^{(8)}$ S.Ar.Go (Articular angle): The angle between the posterior border of ramus and posteriolateral cranial base. ${ }^{(9)}$ N.S.Ar (Saddle angle): The angle between the anterior and posterior cranial base. ${ }^{(9)}$ Sum of posterior angles $(\mathrm{Go}+\mathrm{Ar}+\mathrm{S}){ }^{(9)}$

The data was analyzed by using SPSS program, the analysis include descriptive statistic mean and standard deviation of all linear, ratio and angular measurements of 3 types of overbite. The significant difference of means between males and females for three types of overbite is determined by using t-test at 0.05 level of significance. Analysis of variance (ANOVA) was performed for each variable and Duncan multiple range test was done for testing the significant difference among means.

\section{RESULTS}

The comparisons were described in Tables (1,2,3 and 4). Concerning males and total sample; open, normal and deep overbites show significant difference in TAFH, UAFH and LAFH, but in females, the significant difference is present in TAFH and LAFH. In dental linear measurements, the significant difference is present in UPDH in males, females and total sample, while LPDH is significantly different in the three types of overbite in total sample only. Total sample, males and females show significant difference inside each type of overbite in the following angles: SN-MP, PP-MP, OP-MP, Go angle and summation of posterior angles. However, the total sample shows significant difference in SN-PP angle, while total sample and males show significant difference in OP-PP angle. All skeletal and dental ratios are significantly different in the three types of overbite for total sample, males and females except females in LPDH/LADH ratio, as they showed no significant differences (Table 4). 
Table (1): Comparison of linear skeletal measurements among the three types of overbite for each sex and total sample.

\begin{tabular}{|c|c|c|c|c|c|c|c|c|c|c|c|c|c|}
\hline \multirow{2}{*}{ Variable. } & \multirow{2}{*}{ OB } & \multicolumn{4}{|c|}{ Total sample } & \multicolumn{4}{|c|}{ Male } & \multicolumn{4}{|c|}{ Female } \\
\hline & & Mean & SD & F-value & Sig & Mean & SD & F-value & Sig & Mean & SD & F-value & Sig \\
\hline \multirow{3}{*}{$\begin{array}{l}\text { Total Anterior } \\
\text { Facial Height }\end{array}$} & $\mathrm{O}$ & 128.85B & 7.74 & \multirow{3}{*}{10.0} & \multirow{3}{*}{$\mathrm{S}$} & $130.4 \mathrm{~B}$ & 8.41 & \multirow{3}{*}{5.54} & \multirow{3}{*}{ S } & 127.3B & 6.90 & \multirow{3}{*}{4.86} & \multirow{3}{*}{ S } \\
\hline & $\mathrm{N}$ & $122.94 \mathrm{~A}$ & 6.27 & & & $123.4 \mathrm{~A}$ & 7.14 & & & $122.4 \mathrm{~A}$ & 5.37 & & \\
\hline & $\mathrm{D}$ & $123.85 \mathrm{~A}$ & 7.19 & & & 126.AB & 5.98 & & & $122.4 \mathrm{~A}$ & 7.37 & & \\
\hline \multirow{3}{*}{$\begin{array}{c}\text { Upper Anterior } \\
\text { Facial Height }\end{array}$} & $\mathrm{O}$ & $56.03 \mathrm{~A}$ & 4.06 & \multirow{3}{*}{2.88} & \multirow{3}{*}{ S } & 56.27A & 4.59 & \multirow{3}{*}{3.21} & $\mathrm{~S}$ & $55.80 \mathrm{~A}$ & 3.56 & \multirow{3}{*}{1.02} & \multirow{3}{*}{ NS } \\
\hline & $\mathrm{N}$ & 56.12A & 2.88 & & & $55.66 \mathrm{~A}$ & 3.07 & & & $56.58 \mathrm{~A}$ & 2.66 & & \\
\hline & $\mathrm{D}$ & 57.53B & 3.45 & & & $58.71 \mathrm{~B}$ & 3.79 & & & 56.97A & 3.19 & & \\
\hline \multirow{3}{*}{$\begin{array}{c}\text { Lower Anterior } \\
\text { Facial Height }\end{array}$} & $\mathrm{O}$ & 74.744B & 5.52 & \multirow{3}{*}{24.3} & \multirow{3}{*}{ S } & $76 B$ & 6.15 & \multirow{3}{*}{8.91} & \multirow{3}{*}{ S } & 73.57B & 4.68 & \multirow{3}{*}{15.0} & \multirow{3}{*}{ S } \\
\hline & $\mathrm{N}$ & $68.92 \mathrm{~A}$ & 5.57 & & & $69.76 \mathrm{~A}$ & 6.20 & & & $68.08 \mathrm{~A}$ & 4.85 & & \\
\hline & $\mathrm{D}$ & 67.67A & 5.12 & & & 69.96A & 3.65 & & & $66.58 \mathrm{~A}$ & 5.39 & & \\
\hline \multirow{3}{*}{$\begin{array}{c}\text { Total Posterior } \\
\text { Facial Height }\end{array}$} & $\mathrm{O}$ & 81.14A & 4.94 & \multirow{3}{*}{0.56} & \multirow{3}{*}{ NS } & $82.12 \mathrm{~A}$ & 5.44 & \multirow{3}{*}{0.32} & \multirow{3}{*}{ NS } & $80.23 \mathrm{~A}$ & 4.33 & \multirow{3}{*}{0.65} & \\
\hline & $\mathrm{N}$ & $81.68 \mathrm{~A}$ & 6.34 & & & $82.88 \mathrm{~A}$ & 7.02 & & & $80.48 \mathrm{~A}$ & 5.45 & & NS \\
\hline & $\mathrm{D}$ & 82.39A & 6.28 & & & 83.71A & 5.70 & & & 81.76A & 6.53 & & \\
\hline & $\mathrm{O}$ & $46.12 \mathrm{~A}$ & 3.56 & & & $46.62 \mathrm{~A}$ & 4.39 & & & $45.65 \mathrm{~A}$ & 2.57 & & \\
\hline Facial Height & $\mathrm{N}$ & $44.91 \mathrm{~A}$ & 3.54 & 1.40 & NS & $45.4 \mathrm{~A}$ & 3.97 & 0.62 & NS & $44.42 \mathrm{~A}$ & 3.04 & 0.85 & NS \\
\hline & $\mathrm{D}$ & $45.52 \mathrm{~A}$ & 3.73 & & & $46.18 \mathrm{~A}$ & 2.70 & & & $45.2 \mathrm{~A}$ & 4.12 & & \\
\hline I pren Destarion & $\mathrm{O}$ & $34.1 \mathrm{~A}$ & 3.49 & & & $34.43 \mathrm{~A}$ & 3.03 & & & 33.78A & 3.91 & & \\
\hline Facial Height & $\mathrm{N}$ & $35.86 \mathrm{~A}$ & 5.18 & 2.51 & NS & $36.58 \mathrm{~A}$ & 5.19 & 1.72 & NS & 35.14A & 5.17 & 1.09 & NS \\
\hline & $\mathrm{D}$ & $35.82 \mathrm{~A}$ & 4.58 & & & $36.59 \mathrm{~A}$ & 5.12 & & & $35.45 \mathrm{~A}$ & 4.34 & & \\
\hline & $\mathrm{O}$ & $47.24 \mathrm{~A}$ & 4.20 & & & $47.60 \mathrm{~A}$ & 3.92 & & & 46.90A & 4.49 & & \\
\hline Ramus Height & $\mathrm{N}$ & 48.95A & 4.16 & 2.03 & NS & $48.92 \mathrm{~A}$ & 4.52 & 1.38 & NS & 48.98A & 3.86 & 1.40 & NS \\
\hline & $\mathrm{D}$ & $48.51 \mathrm{~A}$ & 4.81 & & & $49.90 \mathrm{~A}$ & 4.83 & & & $47.85 \mathrm{~A}$ & 4.73 & & \\
\hline
\end{tabular}

OB = Overbite, $\quad \mathrm{O}=$ Open $\quad \mathrm{N}=$ Normal $\quad \mathrm{D}=$ Deep, NS = Not Significant $\mathrm{S}=$ Significant at $P<0.05$.Variables are measured in millimeters. SD: Standard Deviation. Similar letters of A or B means no significant differences, while different letters means significant differences.

Table(2): Comparison of linear dental measurements among the three types of overbite for each sex and total sample.

\begin{tabular}{|c|c|c|c|c|c|c|c|c|c|c|c|c|c|}
\hline \multirow{2}{*}{ Variable. } & \multirow{2}{*}{ OB } & \multicolumn{4}{|c|}{ Total sample } & \multicolumn{4}{|c|}{ Male } & \multicolumn{4}{|c|}{ Female } \\
\hline & & Mean & SD & F-value & Sig & Mean & SD & F-value & Sig & Mean & SD & F-value & Sig \\
\hline \multirow{3}{*}{$\begin{array}{l}\text { Upper Anterior } \\
\text { Dental Height }\end{array}$} & $\mathrm{O}$ & $29.85 \mathrm{~A}$ & 3.48 & \multirow{3}{*}{1.79} & \multirow{3}{*}{ NS } & $30.85 \mathrm{~A}$ & 3.62 & \multirow{3}{*}{2.22} & \multirow{3}{*}{ NS } & $28.92 \mathrm{~A}$ & 3.12 & \multirow{3}{*}{0.56} & \multirow{3}{*}{ NS } \\
\hline & $\mathrm{N}$ & $28.73 \mathrm{~A}$ & 2.83 & & & $29.24 \mathrm{~A}$ & 3.0 & & & $28.22 \mathrm{~A}$ & 2.61 & & \\
\hline & $\mathrm{D}$ & $29.70 \mathrm{~A}$ & 3.28 & & & $31.12 \mathrm{~A}$ & 2.91 & & & 29.02A & 3.27 & & \\
\hline \multirow{3}{*}{$\begin{array}{c}\text { Upper Posterior } \\
\text { Dental Height }\end{array}$} & $\mathrm{O}$ & $24.56 \mathrm{~B}$ & 2.88 & \multirow{3}{*}{13.1} & \multirow{3}{*}{ S } & 24.95B & 3.12 & \multirow{3}{*}{4.28} & \multirow{3}{*}{$S$} & 24.19B & 2.65 & \multirow{3}{*}{9.23} & \multirow{3}{*}{ S } \\
\hline & $\mathrm{N}$ & $22.56 \mathrm{~A}$ & 2.32 & & & $22.86 \mathrm{~A}$ & 2.46 & & & $22.26 \mathrm{~A}$ & 2.18 & & \\
\hline & $\mathrm{D}$ & $22.12 \mathrm{~A}$ & 2.36 & & & $23.4 \mathrm{AB}$ & 1.59 & & & $21.50 \mathrm{~A}$ & 2.43 & & \\
\hline \multirow{3}{*}{$\begin{array}{l}\text { Lower Anterior } \\
\text { Dental Height }\end{array}$} & $\mathrm{O}$ & $44.23 \mathrm{~A}$ & 3.17 & \multirow{3}{*}{1.68} & \multirow{3}{*}{ NS } & $45.37 \mathrm{~A}$ & 3.84 & \multirow{3}{*}{2.10} & \multirow{3}{*}{ NS } & 43.17A & 1.92 & \multirow{3}{*}{0.20} & \multirow{3}{*}{ NS } \\
\hline & $\mathrm{N}$ & 43.09A & 3.16 & & & $43.5 \mathrm{~A}$ & 3.31 & & & $42.68 \mathrm{~A}$ & 3.02 & & \\
\hline & $\mathrm{D}$ & $43.62 \mathrm{~A}$ & 2.97 & & & $44.96 \mathrm{~A}$ & 2.36 & & & 42.98A & 3.04 & & \\
\hline \multirow{3}{*}{$\begin{array}{c}\text { Lower Posterior } \\
\text { Dental Height }\end{array}$} & $\mathrm{O}$ & 34.07B & 2.64 & \multirow{3}{*}{2.62} & \multirow{3}{*}{ S } & $35.02 \mathrm{~A}$ & 2.88 & \multirow{3}{*}{1.78} & \multirow{3}{*}{ NS } & 33.19A & 2.08 & \multirow{3}{*}{0.64} & \multirow{3}{*}{ NS } \\
\hline & $\mathrm{N}$ & 33.3AB & 4.26 & & & 33.66A & 4.57 & & & $33.04 \mathrm{~A}$ & 3.99 & & \\
\hline & $\mathrm{D}$ & $32.57 \mathrm{~A}$ & 2.65 & & & $33.03 \mathrm{~A}$ & 1.87 & & & $32.35 \mathrm{~A}$ & 2.95 & & \\
\hline
\end{tabular}


Table (3):Comparison of angular measurements among the three types of overbite.

\begin{tabular}{|c|c|c|c|c|c|c|c|c|c|c|c|c|c|}
\hline \multirow[b]{2}{*}{ Variable } & \multirow[b]{2}{*}{ OB } & \multicolumn{4}{|c|}{ Total Sample } & \multicolumn{4}{|c|}{ Male } & \multicolumn{4}{|c|}{ Female } \\
\hline & & Mean & SD & $\begin{array}{c}\text { F- } \\
\text { value }\end{array}$ & Sig & Mean & SD & $\begin{array}{c}\text { F- } \\
\text { value }\end{array}$ & Sig & Mean & SD & F-value & Sig \\
\hline \multirow{3}{*}{$\begin{array}{l}\text { Mandible to } \\
\text { anterior cranial } \\
\text { base }\end{array}$} & $\mathrm{O}$ & $38.46 \mathrm{~B}$ & 5.07 & \multirow{3}{*}{21.0} & \multirow{3}{*}{ S } & 38.83B & 4.93 & \multirow{3}{*}{12.8} & \multirow{3}{*}{$S$} & $38.11 B$ & 5.27 & \multirow{3}{*}{11.1} & \multirow{3}{*}{ S } \\
\hline & $\mathrm{N}$ & $32.34 \mathrm{~A}$ & 5.10 & & & $31.16 \mathrm{~A}$ & 4.76 & & & $33.52 \mathrm{~A}$ & 5.24 & & \\
\hline & $\mathrm{D}$ & $32.36 \mathrm{~A}$ & 6.05 & & & $34.18 \mathrm{~A}$ & 6.59 & & & $31.50 \mathrm{~A}$ & 5.68 & & \\
\hline \multirow{3}{*}{$\begin{array}{c}\text { Palato } \\
\text { mandibular } \\
\text { plane angle }\end{array}$} & $\mathrm{O}$ & 30.30B & 4.25 & \multirow{3}{*}{39.7} & \multirow{3}{*}{ S } & 31.06B & 4.08 & \multirow{3}{*}{19.8} & \multirow{3}{*}{ S } & 29.59B & 4.35 & \multirow{3}{*}{20.2} & \multirow{3}{*}{$\mathrm{S}$} \\
\hline & $\mathrm{N}$ & $23.65 \mathrm{~A}$ & 4.87 & & & $23.32 \mathrm{~A}$ & 4.69 & & & 23.98A & 5.11 & & \\
\hline & $\mathrm{D}$ & $22.8 \mathrm{~A}$ & 4.66 & & & $24.25 \mathrm{~A}$ & 5.07 & & & $22.11 \mathrm{~A}$ & 4.36 & & \\
\hline \multirow{3}{*}{$\begin{array}{c}\text { Mandibular } \\
\text { occlusal angle }\end{array}$} & $\mathrm{O}$ & $20.7 \mathrm{~B}$ & 3.94 & \multirow{3}{*}{31.0} & \multirow{3}{*}{ S } & 20.54B & 3.77 & \multirow{3}{*}{14.4} & \multirow{3}{*}{ S } & $20.84 \mathrm{~B}$ & 4.16 & \multirow{3}{*}{17.6} & \multirow{3}{*}{ S } \\
\hline & $\mathrm{N}$ & $15.64 \mathrm{~A}$ & 3.34 & & & $15.28 \mathrm{~A}$ & 3.09 & & & $16.0 \mathrm{~A}$ & 3.59 & & \\
\hline & $\mathrm{D}$ & $15.47 \mathrm{~A}$ & 3.98 & & & $16.59 \mathrm{~A}$ & 3.72 & & & $14.94 \mathrm{~A}$ & 4.04 & & \\
\hline \multirow{3}{*}{$\begin{array}{l}\text { Palatal plane to } \\
\text { anterior cranial } \\
\text { base }\end{array}$} & $\mathrm{O}$ & $8.16 \mathrm{~A}$ & 3.15 & \multirow{3}{*}{2.31} & & $7.75 \mathrm{~A}$ & 3.20 & & & $8.53 \mathrm{~A}$ & 3.12 & & \\
\hline & $\mathrm{N}$ & 8.6AB & 3.44 & & $\mathrm{~S}$ & $7.82 \mathrm{~A}$ & 3.48 & 2.11 & NS & $9.46 \mathrm{~A}$ & 3.27 & 0.73 & NS \\
\hline & $\mathrm{D}$ & $9.52 \mathrm{~B}$ & 3.00 & & & $9.68 \mathrm{~A}$ & 2.74 & & & $9.44 \mathrm{~A}$ & 3.15 & & \\
\hline & $\mathrm{O}$ & $9.59 \mathrm{~B}$ & 3.05 & & & $10.52 \mathrm{~B}$ & 3.26 & & & $8.73 \mathrm{~A}$ & 2.61 & & \\
\hline $\begin{array}{l}\text { Ucciuso paratal } \\
\text { plane angle }\end{array}$ & $\mathrm{N}$ & $7.98 \mathrm{~A}$ & 3.47 & 6.10 & S & $8.04 \mathrm{~A}$ & 3.95 & 4.14 & S & $7.92 \mathrm{~A}$ & 3.00 & 1.87 & NS \\
\hline & $\mathrm{D}$ & $7.29 \mathrm{~A}$ & 3.58 & & & $7.65 \mathrm{~A}$ & 3.38 & & & $7.11 \mathrm{~A}$ & 3.70 & & \\
\hline & $\mathrm{O}$ & $129.6 \mathrm{~B}$ & 6.01 & & & 131.45B & 5.69 & & & $127.8 \mathrm{~B}$ & 5.90 & & \\
\hline Gonial angle & $\mathrm{N}$ & 122.7A & 4.94 & 23.4 & S & $122.68 \mathrm{~A}$ & 4.77 & 18.9 & S & $122.8 \mathrm{~A}$ & 5.21 & 7.87 & S \\
\hline & $\mathrm{D}$ & $122.3 \mathrm{~A}$ & 6.72 & & & $124.03 \mathrm{~A}$ & 5.29 & & & $121.5 \mathrm{~A}$ & 7.24 & & \\
\hline & $\mathrm{O}$ & $146.0 \mathrm{~A}$ & 8.76 & & & 144.39A & 8.99 & & & $147.5 \mathrm{~A}$ & 8.42 & & \\
\hline Articular angle & $\mathrm{N}$ & $145.1 \mathrm{~A}$ & 7.59 & 0.35 & NS & $145.66 \mathrm{~A}$ & 7.67 & 0.22 & NS & $144.6 \mathrm{~A}$ & 7.63 & 1.04 & NS \\
\hline & $\mathrm{D}$ & $146.4 \mathrm{~A}$ & 6.79 & & & $145.87 \mathrm{~A}$ & 6.27 & & & $146.6 \mathrm{~A}$ & 7.10 & & \\
\hline & $\mathrm{O}$ & $123.5 \mathrm{~A}$ & 6.42 & & & $123.95 \mathrm{~A}$ & 5.67 & & & $123.0 \mathrm{~A}$ & 7.13 & & \\
\hline Saddle angle & $\mathrm{N}$ & $124.8 \mathrm{~A}$ & 5.74 & 0.86 & NS & $123.28 \mathrm{~A}$ & 5.99 & 0.42 & NS & $126.4 \mathrm{~A}$ & 5.12 & 1.80 & NS \\
\hline & $\mathrm{D}$ & $124.9 \mathrm{~A}$ & 5.90 & & & $124.93 \mathrm{~A}$ & 4.89 & & & $124.8 \mathrm{~A}$ & 6.39 & & \\
\hline Cummotion & $\mathrm{O}$ & 399.1B & 5.21 & & & 399.81C & 4.75 & & & 398.5B & 5.63 & & \\
\hline $\begin{array}{l}\text { angles } \\
\text { and }\end{array}$ & $\mathrm{N}$ & 392.7A & 5.71 & 18.8 & S & 391.62A & 4.72 & 16.4 & $S$ & 393.8A & 6.46 & 6.53 & S \\
\hline & $\mathrm{D}$ & 393.6A & 5.99 & & & 394.84B & 5.79 & & & $393.1 \mathrm{~A}$ & 6.09 & & \\
\hline
\end{tabular}


Table (4):Comparison of ratios among three types of overbite for each sex and total sample.

\begin{tabular}{|c|c|c|c|c|c|c|c|c|c|c|c|c|c|}
\hline \multirow{2}{*}{ Variables } & \multirow{2}{*}{$\begin{array}{l}\mathbf{O} \\
\mathbf{B}\end{array}$} & \multicolumn{4}{|c|}{ Total Sample } & \multicolumn{4}{|c|}{ Male } & \multicolumn{4}{|c|}{ Female } \\
\hline & & Mean & SD & F-value & Sig. & Mean & SD & F-value & Sig & Mean & SD & F-value & Sig \\
\hline \multirow{3}{*}{$\begin{array}{l}\text { Ratio between } \\
\text { total posterior } \\
\text { and anterior } \\
\text { facial height }\end{array}$} & $\mathrm{O}$ & $0.630 \mathrm{~A}$ & 0.036 & & & $0.630 \mathrm{~A}$ & 0.034 & & & $0.630 \mathrm{~A}$ & 0.039 & & \\
\hline & $\mathrm{N}$ & 0.664B & 0.039 & 11.1 & S & $0.671 \mathrm{~B}$ & 0.038 & 7.16 & S & 0.657B & 0.039 & 5.25 & S \\
\hline & $\mathrm{D}$ & $0.665 B$ & 0.049 & & & $0.660 \mathrm{~B}$ & 0.044 & & & $0.668 B$ & 0.052 & & \\
\hline \multirow{3}{*}{$\begin{array}{c}\text { Ratio between } \\
\text { lower and total } \\
\text { anterior facial } \\
\text { height }\end{array}$} & $\mathrm{O}$ & $0.579 \mathrm{C}$ & 0.019 & & & $0.582 \mathrm{C}$ & 0.020 & & & 0.577B & 0.018 & & \\
\hline & $\mathrm{N}$ & 0.559B & 0.024 & 28.6 & S & $0.564 \mathrm{~B}$ & 0.024 & 10.6 & $\mathrm{~S}$ & $0.555 \mathrm{~A}$ & 0.024 & 16.4 & S \\
\hline & $\mathrm{D}$ & $0.544 \mathrm{~A}$ & 0.026 & & & $0.546 \mathrm{~A}$ & 0.028 & & & $0.543 \mathrm{~A}$ & 0.025 & & \\
\hline \multirow{3}{*}{$\begin{array}{c}\text { Ratio between } \\
\text { upper and total } \\
\text { anterior facial } \\
\text { height }\end{array}$} & $\mathrm{O}$ & $0.434 \mathrm{~A}$ & 0.018 & & & $0.431 \mathrm{~A}$ & 0.021 & & & $0.437 \mathrm{~A}$ & 0.015 & & \\
\hline & $\mathrm{N}$ & $0.460 \mathrm{~B}$ & 0.034 & 20.0 & S & $0.458 \mathrm{~B}$ & 0.045 & 6.15 & S & $0.461 \mathrm{~B}$ & 0.018 & 16.9 & S \\
\hline & $\mathrm{D}$ & $0.464 \mathrm{~B}$ & 0.020 & & & $0.462 B$ & 0.018 & & & $0.465 B$ & 0.022 & & \\
\hline \multirow{3}{*}{$\begin{array}{l}\text { Ratio between } \\
\text { upper posterior } \\
\text { and anterior } \\
\text { dental height }\end{array}$} & $\mathrm{O}$ & $0.825 \mathrm{C}$ & 0.076 & & & $0.810 \mathrm{~B}$ & 0.069 & & & 0.839C & 0.081 & & \\
\hline & $\mathrm{N}$ & $0.786 \mathrm{~B}$ & 0.061 & 17.1 & S & $0.78 \mathrm{AB}$ & 0.060 & 3.59 & S & $0.790 \mathrm{~B}$ & 0.062 & 14.2 & S \\
\hline & $\mathrm{D}$ & $0.747 \mathrm{~A}$ & 0.061 & & & $0.755 \mathrm{~A}$ & 0.056 & & & $0.742 \mathrm{~A}$ & 0.064 & & \\
\hline \multirow{3}{*}{$\begin{array}{l}\text { Ratio between } \\
\text { lower posterior } \\
\text { and anterior } \\
\text { dental height }\end{array}$} & $\mathrm{O}$ & $0.770 \mathrm{~B}$ & 0.046 & & & $0.772 B$ & 0.049 & & & $0.768 \mathrm{~A}$ & 0.045 & & \\
\hline & $\mathrm{N}$ & $0.772 B$ & 0.060 & 4.24 & S & $0.771 \mathrm{~B}$ & 0.067 & 3.01 & S & $0.772 \mathrm{~A}$ & 0.054 & 1.63 & NS \\
\hline & $\mathrm{D}$ & $0.746 \mathrm{~A}$ & 0.038 & & & $0.734 \mathrm{~A}$ & 0.028 & & & $0.752 \mathrm{~A}$ & 0.041 & & \\
\hline
\end{tabular}

OB = Overbite, $\mathrm{O}$ = Open, $\mathrm{N}=$ Normal, $\mathrm{D}=$ Deep, NS = Not Significant, $\mathrm{S}=$ Significant at $P<0.05$. SD: Standard Deviation. Similar letters of A or B means no significant differences, while different letters means significant differences.

\section{DISCUSSIONS}

Except for females, UAFH shows a significant difference of a large value between deep bite group on one hand, and normal and open bite groups on the other hand. Yet there are no significant differences between normal and open bite groups. The findings of this study agree with Nanda, who show that UAFH in deep bite group is greater than open bite group. Open bite group is significantly higher than the other two groups for LAFH, at a time when the last two groups show no significant difference in this respect. The increase of TAFH in open bite group reflects their increased LAFH. ${ }^{(14)}$ Concerning TPFH, UPFH, LPFH, and RH, this study shows that there are no significant differences between the three types of overbite for both sexes and the total sample. The results of this study is in agreement with Beane etal., ${ }^{(14,15)}$ which state that there are no significant differences between open bite and normal bite groups concerning TPFH and $\mathrm{RH}$.

The UPDH is significantly different with a higher value for open bite group than the other two groups, which show no significant differences in this respect (in females and total sample). This increase of UPDH in open bite subjects reveals an over eruption of maxillary first molars in these groups. ${ }^{(16)}$ Concerning LPDH, this study shows that only the total sample is significantly different between open and deep bite groups with no significant difference observed between normal bite and other two bite groups. This means that the mandibular first molars are extruded in open bite subjects. There are no significant differences in upper and lower incisal height between the three types of overbite 
for the total sample and for both sexes. This means that there is no significant difference in the development of the upper and lower incisor region.

SN-MP in the open bite group is significantly higher than the other two bite groups which show no significant differences between them. ${ }^{(14)}$ PP-MP and OP-MP angles also show a significant higher value of open bite subjects than the other two bite groups. which seems to be due to a downward tipping of the mandibular plane. ${ }^{(14)}$ Gonial angle and the summation of the posterior angles also show significant higher value of open bite than the other two bite groups which do not show significant differences in between. ${ }^{(5)}$ SN-PP angle in deep bite group is significantly higher than that in the open bite group in total sample, while no significant difference was seen between the normal bite and the other two bite groups. For this reason, the UAFH in the deep bite group is significantly higher than the other two bite groups. ${ }^{(5,17)}$ OP-PP angle in the open bite group is significantly higher than the other two bite groups. ${ }^{(18)}$ The present study shows no significant differences of Ar and $\mathrm{S}$ angles between the different overbite groups. ${ }^{(17)}$ It can be said that OP-PP angle has greater effect on the overbite opposite to $\mathrm{Ar}$ and $\mathrm{S}$ angles which showed no greater effect on the overbite.

TPFH/TAFH ratio in open bite group is significantly smaller than the other two bite groups for both sexes and total sample due to the increase of TAFH in open bite group. $^{(5,14)} \mathrm{LAFH} / \mathrm{TAFH}$ ratio is significantly different among the different types of overbite with the open bite having larger and the deep bite having smaller values. ${ }^{(12)}$ We can said that the increase of LAFH in open bite subjects leads to an increase of LAFH/TAFH ratio in these subjects. UAFH/TAFH ratio, on the other hand, also shows a significant difference of a smaller value between the open bite and the other two bite groups. This is due to the decrease in UAFH measurement in the open bite subjects. ${ }^{(15)}$ UPDH/UADH ratio is significantly different between the different types of overbite as the open bite group having larger and deep bite group having smaller values. ${ }^{(12)}$ LPDH/LADH ratio shows a significant smaller value of deep bite group than the other two groups (in males and total sample) as the latter show no significant differences in between. ${ }^{(12)}$

\section{CONCLUSION}

Most of the differences between the three types of overbite are seen between open bite, on one hand, and normal and deep overbite on the other hand. The study concludes that open bite group possesses higher values of TAFH, LAFH, UPDH and LPDH, while deep bite group possesses higher value of UAFH.

\section{REFERENCES}

1. Schudy FF. Vertical growth versus antero-posterior growth as related to function and treatment. Angle Orthod.1964; 34(1): 75-8.

2. Atherton JD. The influence of the face height upon the incisor occlusion and lip posture. Dent Practit. 1965; 15(6): 227-231.

3. Pancherz H, Zifber K, Hoyer B. Cephalometric characteristics of class II division 1 and class II division II mal-occlusion: A comparative study in children. Angle Orthod. 1997; 67(2): 111-120.

4. Ceylan I, Eroz B. The effects of overbite on the maxillary and mandibular morphology. Angle Orthod. 2001; 71 (2): 110-115.

5. Cangialosi TJ.Skeletal morphologic features of anterior open bite.Am.J. Orthod. Dentofac. Orthop. 1984; 85(1): 28-36.

6. Major PW, Johnson DE, Hesse KL, Glover, KE. Effect of head orientation on posterior anterior cephalometric landmark identification. Angle Orthod.1996;66(1): 51-60.

7. Tsai HH. Cephalometric studies of children with long and short faces. $J$. Clinical Pediatr.Dent.2000; 25(1): 2328.

8. Tsai HH. Cephalometric characteristics of bimaxillary dentoalveolar protrusion in early mixed dentition. J. Clinical Pediatr. Dent. 2002;26(4): 363-370. 
9. Rakosi T. An atlas and manual of cephalometric radiography. $2^{\text {nd }}$ ed. Wolf Medical Publications LTD.1982;PP.130-148.

10. Bigger staff RH, Allen RC, Tuncay OC, Berkowitz J. A vertical cephalometric analysis of the human craniofacial complex. Am. J. Orthod. 1977; 72(4): 397-405.

11. Fields HW, Donald WW, Keith B , Phillips CL. Relationship between vertical dentofacial morphology and respiration in adolescents. Am.J.Orthod.Dentofac.Orthop.1991; 99(2): 147-54.

12. Fields HW, Proffit WR, Nixon WL, Phillips C, Stanek E. Facial pattern differences in long faced children and adults. Am. J. Orthod. 1984; 85(3): 217-23.

13. Isaacson RJ, Isaacson RJ, Speidel TM, Worms, FW. Extreme variation in vertical facial growth and associated variation in skeletal and dental relations. Angle orthod. 1971;41(4): 219-229.
14. Beane RA, Reimann G, Phillips C, Tulloch C. A cephalometric comparison of black open-bite subjects and black normals. Angle Orthod. 2003;73(3): 294-300.

15. Nanda SK. Patterns of vertical growth in the face. Am. J. Orthod. Dentofac. Orthop.1988; 93(2): 101-6.

16. Haralabakis NB, Yiagtzis SC, Toutountzakis NM. Cephalometric characteristics of open bite in adults: A three dimensional cephalometric evaluation. Int. J. Adult Orthog. Surg.1994; 9(3): 223-231.

17. Nanda SK. Growth patterns in subjects with long and short faces. Am. J. Orthod. Dentofac. Orthop.1990; 98(3): 247-58.

18. Gavito GL, Wallen TR, Little RM, Joondeph DR. Anterior open bite malocclusion. A longitudinal 10-years postretention evaluation of orthodontically treated patients. Am.J. Orthod. Dentofac. Orthop.1985; 87(3): 175-186. 